\title{
GENERAL THEOREMS ON AUTOMORPHISMS OF SEMIGROUPS AND THEIR APPLICATIONS
}

\author{
JOÃO ARAÚJO and JANUSZ KONIECZNY ${ }^{凶}$
}

(Received 8 September 2007; accepted 30 September 2008)

\author{
Communicated by M. G. Jackson
}

\begin{abstract}
We introduce the notion of a strong representation of a semigroup in the monoid of endomorphisms of any mathematical structure, and use this concept to provide a theoretical description of the automorphism group of any semigroup. As an application of our general theorems, we extend to semigroups a wellknown result concerning automorphisms of groups, and we determine the automorphism groups of certain transformation semigroups and of the fundamental inverse semigroups.
\end{abstract}

2000 Mathematics subject classification: primary 20M15; secondary 20M20, 20M30.

Keywords and phrases: automorphism, semigroup, monoid of endomorphisms, representation.

\section{Introduction}

Investigation of automorphism groups of mathematical structures is one of the classical algebraic problems beginning with the works of Evariste Galois. Recently the subject has attracted renewed attention owing to its links with universal algebraic geometry (see, for example, [19]). This paper is concerned with the automorphism group $\operatorname{Aut}(S)$, where $S$ is any semigroup.

A significant amount of research has been devoted to the study of $\operatorname{Aut}(S)$ in the case when $S=\operatorname{End}(\mathcal{A})$ is the monoid of endomorphisms of a mathematical structure $\mathcal{A}$. (A definition of a mathematical structure and its monoid of endomorphisms is provided in Section 2.1.) For example, Schreier [21] and Mal'cev [16] described all automorphisms of $\operatorname{End}(X)$, where $X$ is a set, and Gluskinn [8] described the automorphisms of $\operatorname{End}(V)$, where $V$ is a vector space. More examples are provided by, among others, Formanek [7], Levi [11, 12], Liber [14], Magill [15], Mashevitzky and Schein [17], Schein [20], Sullivan [23], and Šutov [24]. The present authors have

The first author was partially supported by FCT and FEDER, Project POCTI-ISFL-1-143 of Centro de Algebra da Universidade de Lisboa, and by FCT and PIDDAC through the project PTDC/MAT/69514/2006.

(C) 2009 Australian Mathematical Publishing Association Inc. 1446-7887/2009 \$16.00 
contributed to this line of research by studying the automorphism groups of $\operatorname{End}(\mathcal{A})$ for the cases when $\mathcal{A}=(X, \rho, R)$, where $X$ is a set, $\rho$ is an equivalence relation on $X$, and $R$ is a cross section of $X / \rho$ (see [3]); $\mathcal{A}=B \mathcal{V}(X)$, where $X$ is a set, $\mathcal{V}$ is a variety of bands, and $B_{\mathcal{V}}(X)$ is the free object in $\mathcal{V}$ on $X$ (see [4]); $\mathcal{A}=\mathcal{A}_{\mathcal{V}}(X)$, where $X$ is a set, $\mathcal{V}$ is a variety of universal algebras, and $\mathcal{A}_{\mathcal{V}}(X)$ is the 1-simple free algebra in $\mathcal{V}$ on $X$ (see [5]); and $\mathcal{A}=D$, where $D$ is a reflexive digraph [2].

In this paper, we provide a general theorem that may be helpful in describing $\operatorname{Aut}(S)$ for various types of semigroups $S$. Our approach is as follows. Let $\mathcal{A}$ be a mathematical structure. We define a strong representation $\theta: S \rightarrow \operatorname{End}(\mathcal{A})$, and show how the automorphisms of $S$ can be constructed using $\theta$ and automorphisms of $\mathcal{A}$. We then apply the general theorems to particular types of semigroups.

In Section 2, we develop a general theory to find the automorphisms of a semigroup $S$. The main results are Theorem 2.16, where we describe the automorphisms of $S$, and Theorems 2.23 and 2.24, where we characterize the group $\operatorname{Aut}(S)$. In Section 3, we provide applications of our general results. We extend to semigroups a well-known result concerning automorphisms of groups (Theorem 3.1), and we determine $\operatorname{Aut}(S)$ for certain transformation semigroups on a set $X$ (Theorem 3.4), and for the fundamental inverse semigroups (Theorems 3.7 and 3.10).

\section{General theory}

2.1. Preliminaries Let $A$ and $B$ be sets. We denote by $T(A, B)$ the set of all functions from $A$ to $B$ (that is, functions whose domain is $A$ and whose image is a subset of $B$ ). We write functions on the right and compose from left to right, that is, for $f: A \rightarrow B, g: B \rightarrow C$, and $x \in A$, we write $x f$ (not $f(x)$ ) and $x(f g)=(x f) g$ (not $(g f)(x)=g(f(x)))$. We denote $T(A, A)$ by $T(A)$ and note that $T(A)$ is a monoid under function composition. We denote the symmetric group on a set $A$ (which is the group of units of the monoid $T(A))$ by $\operatorname{Sym}(A)$.

We make the concepts of a mathematical structure and its endomorphisms precise through the notion of a concrete category.

Definition 2.1. Let $\mathrm{V}$ be the class of all sets. Following [9, Definition 2.1], we define a concrete category as a triple $C=(\mathrm{Ob}, \mu$, hom $)$, where $\mathrm{Ob}$ is a class and $\mu: \mathrm{Ob} \rightarrow \mathrm{V}$ and hom $: \mathrm{Ob} \times \mathrm{Ob} \rightarrow \mathrm{V}$ are functions such that for all $\mathcal{A}, \mathcal{B}, \mathcal{C} \in \mathrm{Ob}:$

(1) $\operatorname{hom}(\mathcal{A}, \mathcal{B}) \subseteq T(\mathcal{A} \mu, \mathcal{B} \mu)$;

(2) $1_{\mathcal{A} \mu} \in \operatorname{hom}(\mathcal{A}, \mathcal{A})$; and

(3) if $f \in \operatorname{hom}(\mathcal{A}, \mathcal{B})$ and $g \in \operatorname{hom}(\mathcal{B}, \mathcal{C})$, then $\operatorname{fg} \in \operatorname{hom}(\mathcal{A}, \mathcal{C})$.

Elements of $\mathrm{Ob}$ are called objects. For an object $\mathcal{A}$, the set $\mathcal{A} \mu$ is called the underlying set of $\mathcal{A}$.

For example, the category of groups is the triple $(\mathrm{Ob}, \mu$, hom), where $\mathrm{Ob}$ is the class of groups, $\mathcal{A} \mu$ is the underlying set of the group $\mathcal{A}$, and $\operatorname{hom}(\mathcal{A}, \mathcal{B})$ is the set of group homomorphisms from $\mathcal{A}$ to $\mathcal{B}$. The category of topological spaces is the 
triple ( $\mathrm{Ob}, \mu$, hom), where $\mathrm{Ob}$ is the class of topological spaces, $\mathcal{A} \mu$ is the underlying set of the topological space $\mathcal{A}$, and $\operatorname{hom}(\mathcal{A}, \mathcal{B})$ is the set of continuous functions from $\mathcal{A}$ to $\mathcal{B}$.

Definition 2.2. We define a mathematical structure as an object $\mathcal{A}$ of any concrete category. An endomorphism of $\mathcal{A}$ is any element of $\operatorname{hom}(\mathcal{A}, \mathcal{A})$. We denote $\operatorname{hom}(\mathcal{A}, \mathcal{A})$ by $\operatorname{End}(\mathcal{A})$ and note that, by Definition $2.1, \operatorname{End}(\mathcal{A})$ is a submonoid of $T(\mathcal{A} \mu)$. An automorphism of $\mathcal{A}$ is any unit in the monoid $\operatorname{End}(\mathcal{A})$. We denote the group of automorphisms of $\mathcal{A}$ by $\operatorname{Aut}(\mathcal{A})$. Note that an endomorphism $\chi$ of $\mathcal{A}$ is an automorphism of $\mathcal{A}$ if and only if $\chi \in \operatorname{Sym}(\mathcal{A} \mu)$ and $\chi^{-1} \in \operatorname{End}(\mathcal{A})$.

We use the same symbol, say $\mathcal{A}$, both for a mathematical structure $\mathcal{A}$ and for its underlying set $\mathcal{A} \mu$. For example, we write $a \in \mathcal{A}$ meaning $a \in \mathcal{A} \mu$.

Definition 2.3. Let $S$ be a semigroup and $\mathcal{A}$ be a mathematical structure. A representation of $S$ in $\operatorname{End}(\mathcal{A})$ is a homomorphism $\theta: S \rightarrow \operatorname{End}(\mathcal{A})$. If $S$ is a monoid with identity 1 , we require that the homomorphism $\theta$ map 1 to the identity of $\operatorname{End}(\mathcal{A})$.

For $a \in \mathcal{A}$ and $s \in S$, we denote $a(s \theta)$, the image of $a$ under the endomorphism $s \theta \in \operatorname{End}(\mathcal{A})$, by $a \cdot s$. From the fact that $\theta$ is a homomorphism, it follows that $(a \cdot s) \cdot t=a \cdot(s t)$ for all $a \in \mathcal{A}$ and $s, t \in S$. Moreover, if $S$ has an identity 1 , then $a \cdot 1=a$ for all $a \in \mathcal{A}$.

Definition 2.4. We say that a representation $\theta: S \rightarrow \operatorname{End}(\mathcal{A})$ is strong if:

(1) $\theta$ is one-to-one (or faithful);

(2) for every $\phi \in \operatorname{Aut}(S)$, there is an automorphism $\chi \in \operatorname{Aut}(\mathcal{A})$ such that

$$
(\forall a \in \mathcal{A})(\forall s \in S) \quad(a \chi) \cdot(s \phi)=(a \cdot s) \chi .
$$

The idea of a strong representation was introduced by the first author in [1]. We present two examples of strong representations.

EXAMPLE 2.5. Let $S$ be a semigroup and let $X$ be the underlying set of the monoid $S^{1}$, where $S^{1}$ is the semigroup $S$ with an identity 1 adjoined (if necessary). In the category of sets, $\operatorname{End}(X)=T(X)$ and $\operatorname{Aut}(X)=\operatorname{Sym}(X)$. For $s \in S$, define a function $\rho_{s}: S^{1} \rightarrow S^{1}$ by $x \rho_{s}=x s$ for all $x \in S^{1}$. Thus $\rho_{s} \in T(X)$, and we can define $\theta: S \rightarrow T(X)$ by $s \theta=\rho_{s}$. Then $\theta$ is a representation of $S$ in $T(X)$ since for all $s, t \in S$ and $x \in X$,

$$
x \rho_{s t}=x(s t)=(x s) t=(x s) \rho_{t}=\left(x \rho_{s}\right) \rho_{t}=x\left(\rho_{s} \rho_{t}\right),
$$

and so $(s t) \theta=(s \theta)(t \theta)$. This representation, which is faithful since $1 \in S^{1}$, is called the right regular representation of $S$ (see [10, p. 7]). Note that for all $s \in S$ and $x \in X$,

$$
x \cdot s=x(s \theta)=x \rho_{s}=x s .
$$

We claim that the representation is strong. Indeed, for $\phi \in \operatorname{Aut}(S)$, define $\chi: S^{1} \rightarrow S^{1}$ by $x \chi=x \phi$ if $x \in S$ and $1 \chi=1$. Then $\chi \in \operatorname{Sym}(X)$ since $\phi$ is a bijection. Let $x \in X$ 
and $s \in S$. If $x \in S$, then

$$
(x \chi) \cdot(s \phi)=(x \phi) \cdot(s \phi)=(x \phi)(s \phi)=(x s) \phi=(x s) \chi=(x \cdot s) \chi .
$$

If $x=1$, then

$$
(1 \chi) \cdot(s \phi)=1 \cdot(s \phi)=1(s \phi)=s \phi=s \chi=(1 s) \chi=(1 \cdot s) \chi .
$$

Thus the representation is strong.

Definition 2.6. Let $S$ be a semigroup and let $A$ be a subset of $S$. We say that $A$ separates $S$ if for all $s, t \in S$ with $s \neq t$, there is $a \in A$ such that $a s \neq a t$. We say that $A$ is characteristic in $S$ if for all $\phi \in \operatorname{Aut}(S)$, we have $A \phi \subseteq A$. Observe that if $A$ is characteristic in $S$ and $\phi \in \operatorname{Aut}(S)$, then $\phi^{-1} \in \operatorname{Aut}(S)$ and hence $A \phi^{-1} \subseteq A$. Thus if $A$ is characteristic in $S$ and $\phi \in \operatorname{Aut}(S)$, then $A \phi=A$.

EXAMPLE 2.7. Let $S$ be a semigroup separated by a characteristic ideal $R$. Let $Y$ be the underlying set of $R$. We view $Y$ as a set, so $\operatorname{End}(Y)=T(Y)$ and $\operatorname{Aut}(Y)=$ $\operatorname{Sym}(Y)$. For $s \in S$, define a function $\rho_{s}: R \rightarrow R$ by $y \rho_{s}=y s$ ( $y s \in R$ since $R$ is a right ideal of $S$ ). Thus $\rho_{s} \in T(Y)$, and we can define $\theta: S \rightarrow T(Y)$ by $s \theta=\rho_{s}$. Then $\theta$ is a representation of $S$ in $T(Y)$, and this representation is faithful since $R$ separates $S$. Let $\phi \in \operatorname{Aut}(S)$. Denote by $\left.\phi\right|_{R}$ the restriction of $\phi$ to $R$ and note that $\left.\phi\right|_{R} \in \operatorname{Sym}(Y)$ since $R$ is characteristic in $S$. For all $y \in Y$ and $s \in S$,

$$
\left(\left.y \phi\right|_{R}\right) \cdot(s \phi)=\left(\left.y \phi\right|_{R}\right)(s \phi)=(y \phi)(s \phi)=(y s) \phi=\left.(y s) \phi\right|_{R}=\left.(y \cdot s) \phi\right|_{R} .
$$

Thus the representation is strong.

Henceforth, $S$ is a semigroup, $\mathcal{A}$ is a mathematical structure, and $\theta: S \rightarrow \operatorname{End}(\mathcal{A})$ is a strong representation of $S$ in $\operatorname{End}(\mathcal{A})$.

It can happen that for $\phi \in \operatorname{Aut}(S)$, there is more than one automorphism $\chi \in \operatorname{Aut}(\mathcal{A})$ such that $(a \chi) \cdot(s \phi)=(a \cdot s) \chi$ for all $a \in \mathcal{A}$ and $s \in S$. It is therefore useful to introduce the set

$$
\Omega_{\phi}=\{\chi \in \operatorname{Aut}(\mathcal{A}) \mid(\forall a \in \mathcal{A})(\forall s \in S) \quad(a \chi) \cdot(s \phi)=(a \cdot s) \chi\} .
$$

We need the following two lemmas concerning the sets $\Omega_{\phi}$.

LEMMA 2.8. If $\chi$ belongs to $\Omega_{\phi}$, then $\chi^{-1}$ belongs to $\Omega_{\phi^{-1}}$.

Proof. We want to show that for all $a \in \mathcal{A}$ and $s \in S$,

$$
\left(a \chi^{-1}\right) \cdot s \phi^{-1}=(a \cdot s) \chi^{-1} .
$$

However, this is true since, setting $b=a \chi^{-1}$ and $t=s \phi^{-1}$, we have

$$
(a \cdot s) \chi^{-1}=(b \chi \cdot t \phi) \chi^{-1}=(b \cdot t) \chi \chi^{-1}=b \cdot t=\left(a \chi^{-1}\right) \cdot\left(s \phi^{-1}\right),
$$

where the second equality comes from the fact that $\chi \in \Omega_{\phi}$. 
Lemma 2.9. Let $\phi, \psi \in \operatorname{Aut}(S)$. If $\phi \neq \psi$, then $\Omega_{\phi} \cap \Omega_{\psi}=\emptyset$.

PROOF. We prove the contrapositive. Let $\chi \in \Omega_{\phi} \cap \Omega_{\psi}$. Then for all $a \in \mathcal{A}$ and $s \in S$, $(a \chi) \cdot(s \phi)=(a \cdot s) \chi$. Setting $b=a \chi$, we have

$$
\begin{aligned}
a \cdot\left(s \phi \psi^{-1}\right) & =\left(b \chi^{-1}\right) \cdot\left((s \phi) \psi^{-1}\right) \\
& =(b \cdot s \phi) \chi^{-1} \quad\left(\text { since } \chi^{-1} \in \Omega_{\psi^{-1}}\right) \\
& =(a \chi \cdot s \phi) \chi^{-1} \\
& =(a \cdot s) \chi \chi^{-1} \quad\left(\text { since } \chi \in \Omega_{\phi}\right) \\
& =a \cdot s .
\end{aligned}
$$

Hence, for all $a \in \mathcal{A}$ and $s \in S$, we have $a \cdot\left(s \phi \psi^{-1}\right)=a \cdot s$, which implies $s \phi \psi^{-1}=s$ since $\theta$ is faithful. It follows that $\phi=\psi$.

2.2. A description of the elements of $\operatorname{Aut}(S)$ Let $S$ be a semigroup, $\mathcal{A}$ be a mathematical structure, and $\theta: S \rightarrow \operatorname{End}(\mathcal{A})$ be a strong representation of $S$ in $\operatorname{End}(\mathcal{A})$. We define a mapping that assigns to every automorphism $\chi$ of $\mathcal{A}$ a faithful representation $\theta_{\chi}$ of $S$ in $\operatorname{End}(\mathcal{A})$. This is the main tool in our investigation of the automorphisms of $S$. The purpose of this section is to show that the automorphisms of $S$ can be constructed from automorphisms $\chi$ of $\mathcal{A}$, induced representations $\theta_{\chi}$, and the original representation $\theta$.

Definition 2.10. For all $\chi \in \operatorname{Aut}(\mathcal{A})$ and $s \in S$, define $s_{\chi} \in \operatorname{End}(\mathcal{A})$ by

$$
(\forall a \in \mathcal{A}) \quad a s_{\chi}=\left(a \chi^{-1} \cdot s\right) \chi
$$

The mapping $s_{\chi}$ is an endomorphism of $\mathcal{A}$ since it is the composition of endomorphisms, namely $s_{\chi}=\chi^{-1}(s \theta) \chi$.

Let $\chi \in \operatorname{Aut}(\mathcal{A})$. Denote by $\theta_{\chi}$ the mapping $\theta_{\chi}: S \rightarrow \operatorname{End}(\mathcal{A})$ defined by $s \theta_{\chi}=s_{\chi}$. We call $\theta_{\chi}$ the representation of $S$ in $\operatorname{End}(\mathcal{A})$ induced by $\chi$.

The name of $\theta_{\chi}$ is justified by the following lemma.

LEMMA 2.11. The mapping $\theta_{\chi}$ is a faithful representation of $S$ in $\operatorname{End}(\mathcal{A})$.

PROOF. Let $s, t \in S$ be such that $s \theta_{\chi}=t \theta_{\chi}$. Then for all $a \in \mathcal{A}, a s_{\chi}=a t_{\chi}$, that is, $\left(a \chi^{-1} \cdot s\right) \chi=\left(a \chi^{-1} \cdot t\right) \chi$, which implies $a \chi^{-1} \cdot s=a \chi^{-1} \cdot t$. As $\chi^{-1}$ is onto, it follows that $b \cdot s=b \cdot t$ for every $b \in \mathcal{A}$, and so $s=t$ (since $\theta$ is faithful). Thus $\theta_{\chi}$ is faithful. Further, for all $a \in \mathcal{A}$,

$$
\begin{aligned}
a\left(s_{\chi} t_{\chi}\right) & =\left[\left(a \chi^{-1} \cdot s\right) \chi\right] t_{\chi}=\left[\left(\left(a \chi^{-1} \cdot s\right) \chi\right) \chi^{-1} \cdot t\right] \chi \\
& =\left[\left(a \chi^{-1} \cdot s\right) \cdot t\right] \chi=\left[\left(a \chi^{-1}\right) \cdot s t\right] \chi=a(s t)_{\chi} .
\end{aligned}
$$

Thus $\theta_{\chi}$ is a homomorphism, which concludes the proof. 
REMARK 2.12. We now have multiple representations of $S$ in $\operatorname{End}(\mathcal{A})$ : the original strong representation $\theta$ and, for every $\chi \in \operatorname{Aut}(\mathcal{A})$, the induced representation $\theta_{\chi}$. The notation $a \cdot s$, where $s \in S$ and $a \in \mathcal{A}$, will always mean $a(s \theta)$. For $\theta_{\chi}$, we always write $a\left(s \theta_{\chi}\right)$.

Proposition 2.13. Let $\chi \in \operatorname{Aut}(\mathcal{A})$. Then the following are equivalent:

(1) $\chi \in \Omega_{\phi}$ for some $\phi \in \operatorname{Aut}(S)$;

(2) $S \theta_{\chi}=S \theta$;

(3) $\theta_{\chi} \theta^{-1} \in \operatorname{Aut}(S)$.

Proof. To prove that (1) implies (2), suppose that $\chi \in \Omega_{\phi}$, and let $a \in \mathcal{A}$ and $s \in S$. Then setting $t=s \phi$, we have

$$
a\left(s \theta_{\chi}\right)=a s_{\chi}=\left(a \chi^{-1} \cdot s\right) \chi=\left(a \chi^{-1} \cdot t \phi^{-1}\right) \chi=(a \cdot t) \chi^{-1} \chi=a \cdot t=a \cdot s \phi,
$$

where the fourth equality follows from the fact that $\chi^{-1} \in \Omega_{\phi^{-1}}$ (Lemma 2.8). We proved that $s \theta_{\chi}=(s \phi) \theta$ for every $s \in S$ and, hence, $S \theta_{\chi}=(S \phi) \theta$, implying $S \theta_{\chi}=S \theta$ (as $S \phi=S$ ).

To prove that (2) implies (3), note that if $S \theta_{\chi}=S \theta$, then $\theta_{\chi} \theta^{-1}$ is an automorphism as the composition of two isomorphisms: $\theta_{\chi}: S \rightarrow S \theta_{\chi}=S \theta$ and $\theta^{-1}: S \theta \rightarrow S$.

Finally, to prove that (3) implies (1), suppose that $\theta_{\chi} \theta^{-1} \in \operatorname{Aut}(S)$. We want to show that $\chi \in \Omega_{\theta_{\chi} \theta^{-1}}$, that is, that $(a \chi) \cdot\left(s \theta_{\chi} \theta^{-1}\right)=(a \cdot s) \chi$ for all $a \in \mathcal{A}$ and $s \in S$. Suppose that $a \in \mathcal{A}$ and $s \in S$. Then

$$
a \cdot\left(s \theta_{\chi} \theta^{-1}\right)=a\left(\left(s \theta_{\chi} \theta^{-1}\right) \theta\right)=a\left(s \theta_{\chi}\right)=a s_{\chi},
$$

and so

$$
(a \chi) \cdot\left(s \theta_{\chi} \theta^{-1}\right)=(a \chi) s_{\chi}=\left(a \chi \chi^{-1} \cdot s\right) \chi=(a \cdot s) \chi .
$$

The lemma follows.

Observe that the proof that (3) implies (1) in fact proves that if $\theta_{\chi} \theta^{-1} \in \operatorname{Aut}(S)$, then $\chi \in \Omega_{\theta_{\chi} \theta^{-1}}$. This result is important enough to be stated formally.

Lemma 2.14. Let $\chi \in \operatorname{Aut}(\mathcal{A})$ such that $\theta_{\chi} \theta^{-1} \in \operatorname{Aut}(S)$. Then $\chi \in \Omega_{\theta_{\chi} \theta^{-1}}$.

LEMMA 2.15. If $\chi \in \Omega_{\phi}$ for some $\phi \in \operatorname{Aut}(S)$, then $\phi=\theta_{\chi} \theta^{-1}$.

Proof. By Proposition 2.13, if $\chi \in \Omega_{\phi}$, then $\theta_{\chi} \theta^{-1} \in \operatorname{Aut}(S)$. Thus the result follows from Lemmas 2.14 and 2.9.

Now we can give a description of the automorphisms of $S$.

THEOREM 2.16. Let $S$ be a semigroup, $\mathcal{A}$ be a mathematical structure, and suppose that $\theta: S \rightarrow \operatorname{End}(\mathcal{A})$ is a strong representation of $S$ in $\operatorname{End}(\mathcal{A})$. Then

$$
\operatorname{Aut}(S)=\left\{\theta_{\chi} \theta^{-1} \mid \chi \in \operatorname{Aut}(\mathcal{A}) \text { and } S \theta_{\chi}=S \theta\right\} .
$$


PROOF. The converse containment follows from Proposition 2.13. For the direct containment, let $\phi \in \operatorname{Aut}(S)$. Since $\theta$ is a strong representation, there is some $\chi \in \Omega_{\phi}$. By Lemma 2.15, we have $\phi=\theta_{\chi} \theta^{-1}$, and the proof is complete.

We conclude this section with a lemma that is needed in Section 3.3.2.

Lemma 2.17. Let $\chi \in \operatorname{Aut}(\mathcal{A})$ such that $S \theta_{\chi} \subseteq S \theta$. Then $S \theta \subseteq S \theta_{\chi^{-1}}$.

PROOF. Let $s \theta \in S \theta$. To prove that $S \theta \subseteq S \theta_{\chi^{-1}}$, we need to find $t \in S$ such that $t \theta_{\chi^{-1}}=s \theta$. Since $S \theta_{\chi} \subseteq S \theta$, we see that $s \theta_{\chi} \in S \theta$, that is, $s \theta_{\chi}$ is in the domain of $\theta^{-1}$. Define $t=\left(s \theta_{\chi}\right) \theta^{-1}$. We claim that $t \theta_{\chi^{-1}}=s \theta$. Indeed, for all $a \in \mathcal{A}$, we have

$$
\begin{aligned}
a\left(t \theta_{\chi^{-1}}\right) & \left.=(a \chi \cdot t) \chi^{-1} \quad \text { (by the definition of } \theta_{\chi^{-1}}\right) \\
& =((a \chi)(t \theta)) \chi^{-1} \\
& =\left((a \chi)\left(s \theta_{\chi}\right)\right) \chi^{-1} \quad\left(\text { since } t \theta=s \theta_{\chi}\right) \\
& \left.=\left((a \chi) \chi^{-1} \cdot s\right) \chi \chi^{-1} \quad \text { (by the definition of } \theta_{\chi}\right) \\
& =a \cdot s \\
& =a(s \theta) .
\end{aligned}
$$

The result follows.

2.3. A characterization of the group $\operatorname{Aut}(S)$ Let $S$ be a subsemigroup of a monoid $T$, and let $G$ be a subgroup of $T$ containing the identity of $T$. We define the normalizer $N_{G}(S)$ of $S$ in $G$ and the centralizer $C_{G}(S)$ of $S$ in $G$ by

$$
\begin{gathered}
N_{G}(S)=\left\{g \in G \mid g^{-1} S g=S\right\}, \\
C_{G}(S)=\{g \in G \mid(\forall s \in S) g s=s g\} .
\end{gathered}
$$

It is straightforward from the definition of $N_{G}(S)$ to show that for all $g, h \in N_{G}(S)$, $g h \in N_{G}(S)$ and $g^{-1} \in N_{G}(S)$, that is, $N_{G}(S)$ is a subgroup of $G$. Let $g \in G$. If $g s=s g$ for every $s \in S$, then $g^{-1} s g=s$ for every $s \in S$, and so $g^{-1} S g=S$. Thus $C_{G}(S)$ is a subgroup of $N_{G}(S)$.

Now we go back to our assumption that $S$ is a semigroup, $\mathcal{A}$ is a mathematical structure, and $\theta: S \rightarrow \operatorname{End}(\mathcal{A})$ is a strong representation of $S$ in $\operatorname{End}(\mathcal{A})$.

We write $N(S \theta)$ for $N_{\operatorname{Aut}(\mathcal{A})}(S \theta)$ and $C(S \theta)$ for $C_{\operatorname{Aut}(\mathcal{A})}(S \theta)$, that is,

$$
\begin{gathered}
N(S \theta)=\left\{\chi \in \operatorname{Aut}(\mathcal{A}) \mid \chi^{-1}(S \theta) \chi=S \theta\right\}, \\
C(S \theta)=\{\chi \in \operatorname{Aut}(\mathcal{A}) \mid(\forall s \in S) \chi(s \theta)=(s \theta) \chi\} .
\end{gathered}
$$

In this section, we prove that $C(S \theta)$ is a normal subgroup of $N(S \theta)$ and that $\operatorname{Aut}(S)$ is isomorphic to the quotient group $N(S \theta) / C(S \theta)$.

Recall that for $\chi \in \operatorname{Aut}(\mathcal{A}), \theta_{\chi}$ is the representation of $S$ in $\operatorname{End}(\mathcal{A})$ induced by $\chi$ (see Definition 2.10 and Lemma 2.11). 
LEMMA 2.18. Let $\theta: S \rightarrow \operatorname{End}(\mathcal{A})$ be a strong representation. Then

$N(S \theta)=\left\{\chi \in \operatorname{Aut}(\mathcal{A}) \mid S \theta_{\chi}=S \theta\right\}=\bigcup_{\phi \in \operatorname{Aut}(S)} \Omega_{\phi}=\left\{\chi \in \operatorname{Aut}(\mathcal{A}) \mid \theta_{\chi} \theta^{-1} \in \operatorname{Aut}(S)\right\}$

ProOF. Let $\chi \in \operatorname{Aut}(\mathcal{A})$. Recall that for every $s \in S, s \theta_{\chi}=\chi^{-1}(s \theta) \chi$. Thus

$$
S \theta_{\chi}=\left\{s \theta_{\chi} \mid s \in S\right\}=\left\{\chi^{-1}(s \theta) \chi \mid s \in S\right\}=\chi^{-1}(S \theta) \chi,
$$

and so the first equality follows from (2.1). The second and third equalities follow from Proposition 2.13.

Let $\chi \in N(S \theta)$. We denote $\theta_{\chi} \theta^{-1} \in \operatorname{Aut}(S)$ by $\phi_{\chi}$ :

$$
\phi_{\chi}=\theta_{\chi} \theta^{-1} \in \operatorname{Aut}(S) \text {. }
$$

By Lemmas 2.18 and 2.9, we have that

$$
\phi_{\chi} \text { is the unique } \phi \in \operatorname{Aut}(S) \text { such that } \chi \in \Omega_{\phi} \text {. }
$$

REMARK 2.19. The symbol $\phi$ (perhaps with a subscript) always denotes an automorphism of $S ; \chi$ an automorphism of $\mathcal{A}$; and $\theta$ a representation of $S$ in $\operatorname{End}(\mathcal{A})$. For example, if $\phi_{\chi}, \chi$, and $\theta_{\chi}$ occur in a formula, $\phi_{\chi}$ will be an element of $\operatorname{Aut}(S)$, $\chi$ an element of $\operatorname{Aut}(\mathcal{A})$, and $\theta_{\chi}$ a representation.

Lemma 2.20. Let $\chi, \tau \in N(S \theta)$. Then $\chi \tau \in \Omega_{\phi_{\chi} \phi_{\tau}}$.

PROOF. We want to prove that $(a(\chi \tau)) \cdot\left(s\left(\phi_{\chi} \phi_{\tau}\right)\right)=(a \cdot s)(\chi \tau)$ for all $a \in \mathcal{A}$ and $s \in S$. However, this is true since

$$
\begin{aligned}
(a(\chi \tau)) \cdot\left(s\left(\phi_{\chi} \phi_{\tau}\right)\right) & =((a \chi) \tau) \cdot\left(\left(s \phi_{\chi}\right) \phi_{\tau}\right) \\
& \left.=\left((a \chi) \cdot\left(s \phi_{\chi}\right)\right) \tau \quad \text { (since } \tau \in \Omega_{\phi_{\tau}}\right) \\
& =((a \cdot s) \chi) \tau \quad\left(\text { since } \chi \in \Omega_{\phi_{\chi}}\right) \\
& =(a \cdot s)(\chi \tau) .
\end{aligned}
$$

Hence, $\chi \tau \in \Omega_{\phi_{\chi} \phi_{\tau}}$.

LEMMA 2.21. Let $\chi, \tau \in N(S \theta)$. Then $\phi_{\chi \tau}=\phi_{\chi} \phi_{\tau}$.

Proof. Since $N(S \theta)$ is a subgroup of $\operatorname{Aut}(\mathcal{A})$, we see that $\chi \tau \in N(S \theta)$. By Lemma 2.20, $\chi \tau \in \Omega_{\phi_{\chi} \phi_{\tau}}$. By (2.4), $\chi \tau \in \Omega_{\phi_{\chi \tau}}$ and $\phi_{\chi \tau}$ is the unique $\phi \in \operatorname{Aut}(S)$ such that $\chi \tau \in \Omega_{\phi}$. Thus $\phi_{\chi \tau}=\phi_{\chi} \phi_{\tau}$.

LEMMA 2.22. We have $C(S \theta)=\Omega_{\mathrm{id}_{S}}$. 
PROOF. For every $\chi \in \operatorname{Aut}(\mathcal{A})$,

$$
\begin{aligned}
& \chi \in \Omega_{\mathrm{id}_{S}} \Leftrightarrow(\forall s \in S)(\forall a \in \mathcal{A}) \quad(a \chi) \cdot\left(s\left(\operatorname{id}_{S}\right)\right)=(a \cdot s) \chi \\
& \Leftrightarrow(\forall s \in S)(\forall a \in \mathcal{A}) \quad(a \chi) \cdot s=(a \cdot s) \chi \\
& \Leftrightarrow(\forall s \in S)(\forall a \in \mathcal{A}) \quad(a \chi)(s \theta)=(a(s \theta)) \chi \\
& \Leftrightarrow(\forall s \in S)(\forall a \in \mathcal{A}) \quad a(\chi(s \theta))=a((s \theta) \chi) \\
& \Leftrightarrow(\forall s \in S) \quad \chi(s \theta)=(s \theta) \chi
\end{aligned}
$$

and the result follows by (2.2).

THEOREM 2.23. Let $S$ be a semigroup, $\mathcal{A}$ be a mathematical structure, and suppose that $\theta: S \rightarrow \operatorname{End}(\mathcal{A})$ is a strong representation of $S$ in $\operatorname{End}(\mathcal{A})$. Then

$$
\operatorname{Aut}(S)=\left\{\phi_{\chi} \mid \chi \in N(S \theta)\right\} \cong N(S \theta) / C(S \theta) .
$$

PROOF. By (2.3) and Lemma 2.18, the equality is just a restatement of Theorem 2.16.

To prove the congruence, define $f: N(S \theta) \rightarrow \operatorname{Aut}(S)$ by $\chi f=\phi_{\chi}$. Then $f$ is indeed a mapping from $N(S \theta)$ to $\operatorname{Aut}(S)$ by (2.4). Moreover, $f$ is a group homomorphism by Lemma 2.21, and it is onto since $\theta$ is a strong representation. For every $\chi \in N(S \theta)$,

$$
\chi \in \operatorname{ker}(f) \Leftrightarrow \chi f=\mathrm{id}_{S} \Leftrightarrow \phi_{\chi}=\mathrm{id}_{S} \Leftrightarrow \chi \in \Omega_{\mathrm{id}_{S}} \Leftrightarrow \chi \in C(S \theta),
$$

where the last equivalence is true by Lemma 2.22. Thus $\operatorname{ker}(f)=C(S \theta)$ and so $C(S \theta)$ is a normal subgroup of $N(S \theta)$ and the result follows from the first isomorphism theorem for groups.

It is easy to see that the elements of the quotient group $N(S \theta) / C(S \theta)$ (that is, the cosets of $C(S \theta))$ are the sets $\Omega_{\phi}$, where $\phi \in \operatorname{Aut}(S)$. Thus Theorem 2.23 implies the following result.

THEOREM 2.24. Let $S$ be a semigroup, $\mathcal{A}$ be a mathematical structure, and suppose that $\theta: S \rightarrow \operatorname{End}(\mathcal{A})$ is a strong representation of $S$ in $\operatorname{End}(\mathcal{A})$. Then the following are equivalent:

(1) $\operatorname{Aut}(S) \cong N(S \theta)$;

(2) $C(S \theta)$ is the trivial group;

(3) $\left|\Omega_{\phi}\right|=1$ for every $\phi \in \operatorname{Aut}(S)$;

(4) $\left|\Omega_{\mathrm{id}_{S}}\right|=1$.

\section{Applications}

In this section we show how our general results from Section 2 can be used to determine the automorphism groups of particular types of semigroups. 
3.1. General semigroups Theorem 2.23 describes $\operatorname{Aut}(S)$ for a semigroup $S$ provided that we have a strong representation of $S$ in the monoid of endomorphisms of some mathematical structure $\mathcal{A}$. As we saw in Example 2.5, such a representation is provided by the right regular representation of $S$. This gives us a description that extends a well-known result concerning automorphisms of groups to semigroups.

Let $G$ be a group and let $X$ be the underlying set of $G$. Let $G_{0} \leq \operatorname{Sym}(X)$ be the image of $G$ under the right regular representation of $G$ in $\operatorname{Sym}(X)$. Let $N\left(G_{0}\right)$ and $C\left(G_{0}\right)$ be the normalizer and centralizer of $G_{0}$ in $\operatorname{Sym}(X)$. Then

$$
\operatorname{Aut}(G) \cong N\left(G_{0}\right) / C\left(G_{0}\right)
$$

(see [6, Exercise 2.5.6, p. 45] and [22, Theorem 9.2.3, p. 214]).

Let $S$ be a semigroup and let $X$ be the underlying set of $S^{1}$. Let $S_{0} \leq T(X)$ be the image of $S$ under the right regular representation of $S$ in $T(X)$. Let $N\left(S_{0}\right)$ and $C\left(S_{0}\right)$ be the normalizer and centralizer of $S_{0}$ in $\operatorname{Sym}(X)$. We already observed in Section 2.1 that the right regular representation is a strong representation of $S$ in $T(X)$. Thus Theorem 2.23 gives us the following result.

THEOREM 3.1. Let $S$ be an arbitrary semigroup and let $S_{0}$ be the image of $S$ under the right regular representation of $S$. Then

$$
\operatorname{Aut}(S) \cong N\left(S_{0}\right) / C\left(S_{0}\right)
$$

3.2. Transformation semigroups The purpose of this section is purely illustrative. We show how a well-known description of automorphisms can be derived from our general results to illustrate how these results can be applied to particular cases.

Throughout this section we assume that $S$ is a subsemigroup of $T(X)$, the monoid of full transformations of a set $X$.

For $a \in T(X)$, the rank of $a$, denoted by $\operatorname{rank}(a)$, is the cardinality of the image of $a$. An element of $T(X)$ of rank one whose image is $\{x\}$ is denoted by $[x]$.

Let $J_{1}(S)=\{s \in S \mid \operatorname{rank}(s)=1\}$. Suppose that $J_{1}(S) \neq \emptyset$. Then $J_{1}(S)$ is the kernel of $S$ (the minimum ideal of $S$ ), see [10, p. 68], and so it is characteristic in $S$. Let

$$
A=\{x \in X \mid[x] \in S\} .
$$

Note that $A$ is invariant under $S$, that is, for every $s \in S, A s \subseteq A$. (Indeed, if $[x] \in S$ and $s \in S$, then $[x] s=[x s]$, so $x s \in A$.)

Let $\mathcal{A}$ be the set $A$, so $\operatorname{End}(\mathcal{A})=T(A)$ and $\operatorname{Aut}(\mathcal{A})=\operatorname{Sym}(A)$. Define the mapping $\theta: S \rightarrow T(A)$ by $s \theta=\left.s\right|_{A}$. This is well defined since $A$ is invariant under $S$. Note that for all $x \in A$ and $s \in S, x \cdot s=x s$. We prove that $\theta$ is a strong representation provided that $J_{1}(S)$ separates $S$. (Note that if $J_{1}(S)$ separates $S$, then it must be nonempty.)

Lemma 3.2. Suppose that $J_{1}(S)$ separates $S$. Then $\theta: S \rightarrow T(A)$ defined by $s \theta=$ $\left.S\right|_{A}$ is a strong representation of $S$ in $T(A)$. 
PROOF. It is clear that $\theta$ is a homomorphism. Moreover, it is one-to-one since $J_{1}(S)$ separates $S$. Now we want to show that $\theta$ is strong, that is, for every $\phi \in \operatorname{Aut}(S)$, there is $g \in \operatorname{Sym}(A)$ such that for every $x \in A,(x g) \cdot(s \phi)=(x \cdot s) g$. Let $\phi \in \operatorname{Aut}(S)$ and define $g: A \rightarrow A$ by

$$
x g=y \Leftrightarrow[x] \phi=[y] .
$$

Since $\phi$ is a permutation of $J_{1}(S)$, we have $g \in \operatorname{Sym}(A)$. Now, for all $s \in S$ and $x \in A$,

$$
[x g](s \phi)=([x] \phi)(s \phi)=([x] s) \phi=[x s] \phi=[(x s) g],
$$

which implies

$$
(x g) \cdot(s \phi)=(x g)(s \phi)=(x g)([x g](s \phi))=(x g)[(x s) g]=(x s) g=(x \cdot s) g .
$$

The result follows.

LEMMA 3.3. Suppose that $J_{1}(S)$ separates $S$. Then $\left|\Omega_{\mathrm{id}_{S}}\right|=1$.

PROOF. Let $g \in \Omega_{\mathrm{id}_{S}}$. Then for all $x \in A$ and $s \in S$, we have $(x g) \cdot\left(s\left(\operatorname{id}_{S}\right)\right)=$ $(x \cdot s) g$, that is, $(x g) s=(x s) g$. Taking $s=[x]$, we obtain $(x g)[x]=(x[x]) g$, that is, $x=x g$. We proved that if $g \in \Omega_{\mathrm{id}_{S}}$, then $g=\mathrm{id}_{A}$. The lemma follows.

Recall that the normalizer of $S \theta$ in $\operatorname{Sym}(A)$ (see (2.1)) is the group

$$
N(S \theta)=\left\{g \in \operatorname{Sym}(A) \mid g^{-1}(S \theta) g=S \theta\right\} .
$$

In our particular case,

$$
S \theta=\left\{\left.s\right|_{A} \mid s \in S\right\} .
$$

Since $A$ is invariant under $S, g^{-1} s g=g^{-1}\left(\left.s\right|_{A}\right) g$ for all $s \in S$ and $g \in \operatorname{Sym}(A)$. Thus

$$
N(S \theta)=\left\{g \in \operatorname{Sym}(A) \mid g^{-1} S g=S \theta\right\} .
$$

By Lemma 3.3 and Theorem 2.24, we obtain the following theorem.

THEOREM 3.4. Let $S$ be a subsemigroup of $T(X)$ such that $J_{1}(S)$ separates $S$, and let $A$ be the set defined in (3.1). Then $\operatorname{Aut}(S) \cong N(S \theta)$.

Theorem 3.4 generalizes a well-known result. For $g \in N_{\operatorname{Sym}(X)}(S)$, define $\phi^{g}$ : $S \rightarrow S$ by $s \phi^{g}=g^{-1} s g$. The mapping $\phi^{g}$ is an automorphism of $S$, called the inner automorphism induced by $g$ (see [23]). Let $g \in N(S \theta)$. Recall that $\theta_{g}$ is the representation of $S$ in $T(A)$ induced by $g$ (see Definition 2.10) and that $\phi_{g}=\theta_{g} \theta^{-1}$ is the unique $\phi \in \operatorname{Aut}(S)$ such that $g \in \Omega_{\phi}$ (see (2.3) and (2.4)). Now suppose that $A=X$. Then $\theta=\mathrm{id}_{S}, S \theta=S, N(S \theta)=N_{\text {Sym }(X)}(S)$, and for all $g \in N(S \theta)$, $s \phi_{g}=s\left(\theta_{g} \theta^{-1}\right)=s \theta_{g}=g^{-1} s g$, that is, $\phi_{g}=\phi^{g}$. Hence, the following result is a special case of Theorem 3.4.

Corollary 3.5 (Sullivan [23] and Levi and Seif [13]). Let $S$ be subsemigroup of $T(X)$ that contains all constant transformations of $X$. Then

$$
\operatorname{Aut}(S)=\left\{\phi^{g} \mid g \in N_{\operatorname{Sym}(X)}(S)\right\} \cong N_{\operatorname{Sym}(X)}(S) .
$$


3.3. Fundamental inverse semigroups In this section we continue to show how the general results from Section 2 can be used to describe the automorphisms of particular types of semigroups. The main results of this section give the automorphisms for an important class of semigroups, whose automorphisms have not been described before.

A commutative semigroup $E$ of idempotents is called a semilattice. The relation $\leq$ on $E$ defined by $e \leq f$ if $e f=e$ is a partial order on $E$. For all $e, f \in E$, ef is the greatest lower bound of $e$ and $f$ (see [10, Section 1.3]). It is easy to see that for every mapping $\phi: E \rightarrow E, \phi$ is an automorphism of the semigroup $E$ if and only if $\phi$ is an automorphism of the poset $(E, \leq)$.

A semigroup $S$ is called an inverse semigroup if for every $a \in S$, there is exactly one $a^{-1} \in S$ (called the inverse of $a$ ) such that $a=a a^{-1} a$ and $a^{-1}=a^{-1} a a^{-1}$. If $S$ is an inverse semigroup, then the idempotents of $S$ commute, and for all $a, b \in S$, $a a^{-1}$ is an idempotent and $(a b)^{-1}=b^{-1} a^{-1}$. The set $E(S)$ of idempotents of an inverse semigroup $S$ is a semilattice, called the semilattice of idempotents of $S$ (see [10, Chapter 5]).

Let $S$ be an inverse semigroup. Define a relation $\mu$ on $S$ by

$$
\mu=\left\{(a, b) \in S \times S \mid(\forall e \in E(S)) a^{-1} e a=b^{-1} e b\right\} .
$$

The relation $\mu$ is the largest congruence on $S$ that separates idempotents (for all $e, f \in E(S)$, if $(e, f) \in \mu$, then $e=f)$; see [10, p. 160].

An inverse semigroup $S$ is called fundamental if $\mu$ is the identity relation on $S$ (see [10, p. 161]). In what follows, $S$ is a fundamental inverse semigroup and $E(S)$ is its semilattice of idempotents.

We give two descriptions of the group $\operatorname{Aut}(S)$.

3.3.1. First description We take the mathematical structure $\mathcal{A}$ to be the semilattice $E(S)$. Define $\theta: S \rightarrow \operatorname{End}(E(S))$ by

$$
(\forall s \in S)(\forall e \in E(S)) \quad e(s \theta)=s^{-1} e s .
$$

LEMMA 3.6. The mapping $\theta$ defined in (3.3) is a strong representation of $S$ in the monoid $\operatorname{End}(E(S))$.

PROOF. For all $s \in S$ and $e, f \in E(S)$,

$$
\begin{gathered}
\left(s^{-1} e s\right)\left(s^{-1} e s\right)=s^{-1} e\left(s s^{-1}\right) e s=\left(s^{-1} s s^{-1}\right) e e s=s^{-1} e s, \quad \text { and } \\
(e f) \cdot s=s^{-1} e f s=s^{-1} s s^{-1} e f s=s^{-1} e s s^{-1} f s=\left(s^{-1} e s\right)\left(s^{-1} f s\right)=(e \cdot s)(f \cdot s),
\end{gathered}
$$

and so $s \theta \in \operatorname{End}(E(S))$. It follows from (3.2) and the fact that $S$ is fundamental that $\theta$ is one-to-one. Further, $\theta$ is a homomorphism because for all $s, t \in S$ and $e \in E(S)$,

$$
e \cdot(s t)=(s t)^{-1} e(s t)=t^{-1} s^{-1} e s t=t^{-1}\left(s^{-1} e s\right) t=t^{-1}(e \cdot s) t=(e \cdot s) \cdot t,
$$

and so $(s t) \theta=(s \theta)(t \theta)$. 
Let $\phi \in \operatorname{Aut}(S)$. We want to show that there is a $\chi \in \operatorname{Aut}(E(S))$ such that $(e \chi)$. $(s \phi)=(e \cdot s) \chi$ for all $s \in S$ and $e \in E(S)$. Let $\chi=\left.\phi\right|_{E(S)}$. Then $\chi \in \operatorname{Aut}(E(S))$ since $E(S)$ is characteristic in $S$. Let $s \in S$ and $e \in E(S)$. Taking $t=s \phi$,

$$
(e \chi) \cdot(s \phi)=(e \chi) \cdot t=t^{-1}(e \chi) t=\left(s^{-1} \phi\right)(e \chi)(s \phi)=\left.\left(s^{-1} e s\right) \phi\right|_{E(S)}=(e \cdot s) \chi .
$$

This concludes the proof.

Recall that the normalizer of $S \theta$ in $\operatorname{Aut}(E)$ (see (2.1)) is the group

$$
N(S \theta)=\left\{\chi \in \operatorname{Aut}(E) \mid \chi^{-1}(S \theta) \chi=S \theta\right\} .
$$

THEOREM 3.7. Let $S$ be a fundamental inverse semigroup. Then

$$
\operatorname{Aut}(S)=\left\{\phi_{\chi} \mid \chi \in N(S \theta)\right\} \cong N(S \theta) .
$$

PROOF. By Theorems 2.23 and 2.24, we only have to prove that $\left|\Omega_{\mathrm{id}_{S}}\right|=1$. Let $\chi \in \Omega_{\mathrm{id} s}$. Then for all $s \in S$ and $e \in E(S),(e \chi) \cdot\left(s\left(\mathrm{id}_{S}\right)\right)=(e \cdot s) \chi$, that is, $(e \chi) \cdot s$ $=(e \cdot s) \chi$. In particular, when $s=e$ we have

$$
\begin{array}{r}
e \chi=(e e e) \chi=\left(e^{-1} e e\right) \chi=(e \cdot e) \chi=(e \chi) \cdot e=e^{-1}(e \chi) e \\
=e(e \chi) e=e e(e \chi)=e(e \chi),
\end{array}
$$

so $e \chi \leq e$. By Lemma 2.8, $\chi \in \Omega_{\mathrm{id}_{S}}$ implies $\chi^{-1} \in \Omega_{\mathrm{id}_{S}^{-1}}=\Omega_{\mathrm{id}_{S}}$ and hence $e \chi^{-1} \leq e$. Since $\chi$ is an automorphism of $E(S)$, and so an automorphism of $(E(S), \leq)$, we see that $e \chi^{-1} \leq e$ implies

$$
e=\left(e \chi^{-1}\right) \chi \leq e \chi \leq e .
$$

Thus for all $e \in E(S), e \chi=e$, that is, $\chi=\operatorname{id}_{E(S)}$. We have proved that $\Omega_{\mathrm{id}_{S}}=$ $\left\{\operatorname{id}_{E(S)}\right\}$, and the theorem follows.

3.3.2. Second description In this section, we describe the normalizer $N(S \theta)$ in terms of the semigroup $S$ itself rather than its image $S \theta$, with the help of the fundamental inverse semigroup constructed by Munn [18]. Let $E$ be a semilattice. For every $e \in E$, the set $E e=\{f \in E \mid f \leq e\}$ is the principal ideal of $E$ generated by $e$. For all $e, f \in E$ such that $E e \cong E f$, denote by $T_{e, f}$ the set of all isomorphisms from $E e$ to $E f$, and let

$$
T_{E}=\bigcup\left\{T_{e, f} \mid e, f \in E \text { such that } E e \cong E f\right\} .
$$

The set $T_{E}$ is a subset of the symmetric inverse semigroup $\mathcal{I}_{E}$ of all partial one-to-one transformations of $E$. It is in fact an inverse subsemigroup of $\mathcal{I}_{E}$, called the Munn semigroup of $E$. The semilattice of idempotents $E\left(T_{E}\right)$, which we denote by $E_{0}$, is the set

$$
E_{0}=\left\{1_{e} \mid e \in E\right\},
$$

where for every $e \in E, 1_{e}$ is the identity transformation of the ideal $E e$. The semilattice $E_{0}$ is isomorphic to $E$ via the isomorphism $e \mapsto 1_{e}$. 
An inverse subsemigroup $S$ of $T_{E}$ is called full if $E_{0} \subseteq S$. Every full inverse subsemigroup of $T_{E}$ is fundamental. Moreover, every fundamental inverse semigroup with semilattice of idempotent $E$ is isomorphic to a full inverse subsemigroup of $T_{E}$ (see [10, Section 5.4]).

In view of this result, we may assume from now on that our fundamental inverse semigroup $S$ is a full inverse subsemigroup of $T_{E}$, where $E=E(S)$. In this context, the structure $\mathcal{A}$ is the semilattice $E_{0}$.

For $\alpha \in \mathcal{I}_{E}$, we denote by $\operatorname{dom}(\alpha)$ the domain of $\alpha$ and by $\operatorname{im}(\alpha)$ the image of $\alpha$.

LeMma 3.8. For all $t \in T_{E}$ and $\chi \in \operatorname{Aut}(E), \chi^{-1} t \chi \in T_{E}$.

Proof. Let $t: E e \rightarrow E f$ be an element of $T_{E}$. It is an easy exercise to show that

(1) $\operatorname{dom}\left(\chi^{-1} t \chi\right)=(E e) \chi=E(e \chi)$;

(2) $\operatorname{im}\left(\chi^{-1} t \chi\right)=(E f) \chi=E(f \chi)$;

(3) $\chi^{-1} t \chi$ is a homomorphism from $E(e \chi)$ to $E(f \chi)$;

(4) $\chi^{-1} t \chi$ is a bijection.

Thus $\chi^{-1} t \chi$ is an isomorphism from $E(e \chi)$ to $E(f \chi)$, and so an element of $T_{E}$.

For any $\chi \in \operatorname{Aut}(E)$, define $\chi_{0}: E_{0} \rightarrow E_{0}$ by

$$
1_{e} \chi_{0}=1_{e \chi}
$$

Since for all $e, f \in E, 1_{e} 1_{f}=1_{e f}$, it is clear that $\chi_{0} \in \operatorname{Aut}\left(E_{0}\right)$. Moreover, the mapping $\chi \mapsto \chi_{0}$ is an isomorphism from $\operatorname{Aut}(E)$ to $\operatorname{Aut}\left(E_{0}\right)$. It follows from Lemma 3.8 and its proof that for all $e \in E$,

$$
1_{e} \chi_{0}=\chi^{-1} 1_{e} \chi
$$

In this context ( $S$ is a subsemigroup of $T_{E}$, while $E(S)=E_{0}=\left\{1_{e} \mid e \in E\right\}$, and $\mathcal{A}$ is the semilattice $\left.E_{0}\right)$, the strong representation $\theta: S \rightarrow \operatorname{End}\left(E_{0}\right)$ is defined by

$$
(\forall s \in S)(\forall e \in E) \quad 1_{e}(s \theta)=s^{-1} 1_{e} s .
$$

From (2.1), the normalizer $N(S \theta)$ is

$$
N(S \theta)=\left\{\chi_{0} \in \operatorname{Aut}\left(E_{0}\right) \mid \chi_{0}^{-1}(S \theta) \chi_{0}=S \theta\right\} .
$$

LEMMA 3.9. Let $S$ be a full subsemigroup of $T_{E}$ and let $\theta: S \rightarrow \operatorname{End}\left(E_{0}\right)$ be the strong representation defined in (3.5). Then

$$
N(S \theta)=\left\{\chi_{0} \in \operatorname{Aut}\left(E_{0}\right) \mid \chi^{-1} S \chi=S\right\} .
$$

Proof. Define $\theta_{1}: T_{E} \rightarrow \operatorname{End}\left(E_{0}\right)$ by

$$
\left(\forall t \in T_{E}\right)(\forall e \in E) \quad 1_{e}\left(t \theta_{1}\right)=t^{-1} 1_{e} t
$$


and note that $\theta=\left.\left(\theta_{1}\right)\right|_{S}$. Let $\chi \in \operatorname{Aut}(E)$. Recall that $\theta_{\chi_{0}}: S \rightarrow \operatorname{End}\left(E_{0}\right)$ is defined by

$$
(\forall s \in S)(\forall e \in E) \quad 1_{e}\left(s \theta_{\chi_{0}}\right)=\left(1_{e} \chi_{0}^{-1} \cdot s\right) \chi_{0} .
$$

By (3.4) and the fact that $\chi_{0}^{-1}=\left(\chi^{-1}\right)_{0}$, we have for all $s \in S$ and $e \in E$,

$$
\begin{aligned}
1_{e}\left(s \theta_{\chi_{0}}\right) & =\left(1_{e} \chi_{0}^{-1} \cdot s\right) \chi_{0}=\left(s^{-1}\left(1_{e} \chi_{0}^{-1}\right) s\right) \chi_{0}=\chi^{-1}\left(s^{-1}\left(1_{e} \chi_{0}^{-1}\right) s\right) \chi \\
& =\chi^{-1}\left(s^{-1}\left(\chi 1_{e} \chi^{-1}\right) s\right) \chi=\left(\chi^{-1} s \chi\right)^{-1} 1_{e}\left(\chi^{-1} s \chi\right)=1_{e}\left(\left(\chi^{-1} s \chi\right) \theta_{1}\right) .
\end{aligned}
$$

We have proved that, for all $\chi \in \operatorname{Aut}(E)$,

$$
(\forall s \in S) \quad s \theta_{\chi_{0}}=\left(\chi^{-1} s \chi\right) \theta_{1} .
$$

Suppose that $\chi_{0} \in N(S \theta)$. Then by Lemma 2.18, $S \theta_{\chi_{0}}=S \theta$. Let $s \in S$. By (3.6), $s \theta_{\chi_{0}}=\left(\chi^{-1} s \chi\right) \theta_{1}$. Thus $\left(\chi^{-1} s \chi\right) \theta_{1} \in S \theta$, and so there is $s^{\prime} \in S$ such that $\left(\chi^{-1} s \chi\right) \theta_{1}=s^{\prime} \theta$. Hence, since $\theta=\left.\left(\theta_{1}\right)\right|_{s}$, we have $\left(\chi^{-1} s \chi\right) \theta_{1}=s^{\prime} \theta_{1}$, and so $\chi^{-1} s \chi=s^{\prime}$ (since, by Lemma 3.6, $\theta_{1}$ is one-to-one). It follows that $\chi^{-1} S \chi \subseteq S$. Since $\chi_{0} \in N(S \theta)$ implies $\left(\chi^{-1}\right)_{0}=\chi_{0}^{-1} \in N(S \theta)$, we also have $\chi S \chi^{-1} \subseteq S$, and so $\chi^{-1} S \chi=S$, which proves that $N(S \theta) \subseteq\left\{\chi_{0} \in \operatorname{Aut}\left(E_{0}\right) \mid \chi^{-1} S \chi=S\right\}$.

For the reverse containment, suppose that $\chi \in \operatorname{Aut}(E)$ is such that $\chi^{-1} S \chi=S$. Let $s \in S$. Then $\chi^{-1} s \chi \in S$, and so, by (3.6), $s \theta_{\chi_{0}}=\left(\chi^{-1} s \chi\right) \theta_{1}=\left(\chi^{-1} s \chi\right) \theta \in S \theta$. We have proved that $S \theta_{\chi_{0}} \subseteq S \theta$. Since $\chi^{-1} S \chi=S$ implies $\chi S \chi^{-1}=S$, we also have $S \theta_{\chi_{0}^{-1}} \subseteq S \theta$. Thus by Lemma 2.17, $S \theta \subseteq S \theta_{\chi_{0}}$. Hence, $S \theta=S \theta_{\chi_{0}}$, and so $\chi_{0} \in N(S \theta)$ by Lemma 2.18. The proof is complete.

The group $\operatorname{Aut}(E)$ is not necessarily a subgroup of $T_{E}$. However, we have $S \leq T_{E} \leq \mathcal{I}_{E}$ and $\operatorname{Aut}(E)$ is a subgroup of the symmetric inverse semigroup $\mathcal{I}_{E}$. Thus we have the normalizer of $S$ in $\operatorname{Aut}(E)$ :

$$
N_{\operatorname{Aut}(E)}(S)=\left\{\chi \in \operatorname{Aut}(E) \mid \chi^{-1} S \chi=S\right\} .
$$

Every $\chi \in N_{\operatorname{Aut}(E)}(S)$ induces a mapping $\phi^{\chi}: S \rightarrow S$ defined by

$$
s \phi^{\chi}=\chi^{-1} s \chi
$$

It is clear that $\phi^{\chi} \in \operatorname{Aut}(S)$ for every $\chi \in N_{\operatorname{Aut}(E)}(S)$.

THEOREM 3.10. Let $E$ be a semilattice and let $S$ be a full inverse subsemigroup of the Munn semigroup $T_{E}$. Then

$$
\operatorname{Aut}(S)=\left\{\phi^{\chi} \mid \chi \in N_{\operatorname{Aut}(E)}(S)\right\} \cong N_{\operatorname{Aut}(E)}(S) .
$$

Proof. Let $\chi \in N_{\operatorname{Aut}(E)}(S)$. Then $\phi^{\chi} \in \operatorname{Aut}(S)$, and so $\left.\left(\phi^{\chi}\right)\right|_{E_{0}} \in \operatorname{Aut}\left(E_{0}\right)$. Thus there is $\eta \in \operatorname{Aut}(E)$ such that $\left.\left(\phi^{\chi}\right)\right|_{E_{0}}=\eta_{0}$. We claim that $\eta=\chi$. Indeed, for every $e \in E$,

$$
1_{e} \eta_{0}=1_{e} \phi^{\chi}=\chi^{-1} 1_{e} \chi=1_{e} \chi_{0},
$$


which implies $\eta_{0}=\chi_{0}$, and so $\eta=\chi$. Thus $\left.\left(\phi_{\chi}\right)\right|_{E_{0}}=\chi_{0}$ and so, by the proof of Lemma 3.6, $\chi_{0} \in \Omega_{\phi^{\chi}}$, which implies $\phi^{\chi}=\phi_{\chi_{0}}$. Hence, we have

$$
\operatorname{Aut}(S)=\left\{\phi^{\chi} \mid \chi \in N_{\operatorname{Aut}(E)}(S)\right\} \cong N(S \theta)
$$

by Theorem 3.7. The result follows since $N(S \theta) \cong N_{\text {Aut }(E)}(S)$ by Lemma 3.9.

Clearly $\chi^{-1} T_{E} \chi=T_{E}$ for every $\chi \in \operatorname{Aut}(E)$. In other words, $N_{\operatorname{Aut}(E)}\left(T_{E}\right)=\operatorname{Aut}(E)$. Thus Theorem 3.10 gives the following corollary.

Corollary 3.11. Let $E$ be a semilattice and let $T_{E}$ be the Munn semigroup of $E$. Then $\operatorname{Aut}\left(T_{E}\right) \cong \operatorname{Aut}(E)$.

The bicyclic semigroup $B$ is the semigroup defined by the monoid presentation $B=\langle a, b \mid a b=1\rangle$. It is isomorphic to the Munn semigroup $T_{C_{\omega}}$, where

$$
C_{\omega}=\left\{e_{0}, e_{1}, e_{2}, \ldots\right\} \quad \text { and } e_{0}>e_{1}>e_{2}>\cdots
$$

(see [10, p. 163]). As $C_{\omega}$ is a countable chain with a maximal element, we have that $\operatorname{Aut}\left(C_{\omega}\right)=\left\{\operatorname{id}_{C_{\omega}}\right\}$. Thus the following result (which is well known and straightforward) is a special case of Corollary 3.11.

COROLlARY 3.12. The automorphism group of the bicyclic semigroup is trivial.

\section{Acknowledgement}

The authors would like to thank the referee for an outstanding report, whose comments led to a significantly improved and better structured paper.

\section{References}

[1] J. Araújo, 'Aspects of the endomorphism monoids of independence algebras', PhD Thesis, University of York, 2000.

[2] J. Araújo, E. Dobson and J. Konieczny, 'Automorphisms of endomorphism semigroups of reflexive digraphs', Math. Nachr. to appear.

[3] J. Araújo and J. Konieczny, 'Automorphism groups of centralizers of idempotents', J. Algebra 269 (2003), 227-239.

[4] - 'Automorphisms of endomorphism monoids of relatively free bands', Proc. Edinburgh Math. Soc. (2) 50 (2007), 1-21.

[5] , 'Automorphisms of endomorphism monoids of 1-simple free algebras', Comm. Algebra 37 (2009), 83-94.

[6] J. D. Dixon and B. Mortimer, Permutation Groups, Graduate Texts in Mathematics, 163 (Springer, New York, 1996).

[7] E. Formanek, 'A question of B. Plotkin about the semigroup of endomorphisms of a free group', Proc. Amer. Math. Soc. 130 (2002), 935-937.

[8] L. M. Gluskǐn, 'Semigroups and rings of endomorphisms of linear spaces I', Amer. Math. Soc. Transl. 45 (1965), 105-137.

[9] H. Herrlich and G. E. Strecker, Category Theory: An Introduction (Allyn and Bacon, Boston, MA, 1973).

[10] J. M. Howie, Fundamentals of Semigroup Theory (Oxford University Press, New York, 1995). 
[11] I. Levi, 'Automorphisms of normal transformation semigroups', Proc. Edinburgh Math. Soc. (2) 28 (1985), 185-205.

[12] I. Levi, 'Automorphisms of normal partial transformation semigroups', Glasgow Math. J. 29 (1987), 149-157.

[13] I. Levi and S. Seif, 'Finite normal semigroups', Semigroup Forum 57 (1998), 69-74.

[14] A. E. Liber, 'On symmetric generalized groups', Mat. Sbornik N.S. 33 (1953), 531-544; (in Russian).

[15] K. D. Magill, 'Semigroup structures for families of functions, I. Some homomorphism theorems', J. Aust. Math. Soc. 7 (1967), 81-94.

[16] A. I. Mal'cev, 'Symmetric groupoids', Mat. Sbornik N.S. 31 (1952), 136-151; (in Russian).

[17] G. Mashevitzky and B. M. Schein, 'Automorphisms of the endomorphism semigroup of a free monoid or a free semigroup', Proc. Amer. Math. Soc. 131 (2003), 1655-1660.

[18] W. D. Munn, 'Uniform semilattices and bisimple inverse semigroups', Quart. J. Math. Oxford Ser. (2) 17 (1966), 151-159.

[19] E. Rips, 'Preface [Special issue on papers from the Conference in Honor of the 80th Birthday of Professor Boris I. Plotkin]', Internat. J. Algebra Comput. 17 (2007), v-vii.

[20] B. M. Schein, 'Ordered sets, semilattices, distributive lattices and Boolean algebras with homomorphic endomorphism semigroups', Fund. Math. 68 (1970), 31-50.

[21] I. Schreier, 'Über Abbildungen einer abstrakten Menge Auf ihre Teilmengen', Fund. Math. 28 (1936), 261-264.

[22] W. R. Scott, Group Theory (Prentice Hall, Englewood Cliffs, NJ, 1964).

[23] R. P. Sullivan, 'Automorphisms of transformation semigroups', J. Aust. Math. Soc. 20 (1975), $77-84$.

[24] È. G. Šutov, 'Homomorphisms of the semigroup of all partial transformations', Izv. Vyš̌. Učebn. Zaved. Mat. 3 (1961), 177-184; (in Russian).

JOÃO ARAÚJO, Universidade Aberta, R. Escola Politécnica, 147, 1269-001 Lisboa, Portugal

and

Centro de Álgebra, Universidade de Lisboa, 1649-003 Lisboa, Portugal

e-mail: jarauja@ptmat.fc.ul.pt

JANUSZ KONIECZNY, Department of Mathematics, University of Mary Washington, Fredericksburg, VA 22401, USA

e-mail: jkoniecz@umw.edu 